\title{
Navigational Characteristics of E-document Readers
}

\section{Asim Qayyum}

University of Toronto, 140 St. George St. Toronto, ON, Canada, M5S 3G6. asim.qayum@utoronto.ca

\section{Igor Bilykh}

University of Toronto, 140 St. George St. Toronto, ON, Canada, M5S 3G6. igor.bilykh@utoront.ca

The purpose of this study was to examine the navigational patterns of graduate students' text markings when they interact with electronic documents during an active reading process, thus taking on the role of authors. The readings took place in two settings, pri-vate and document sharing, where in the latter environment each document was shared among a group of students. The resulting interaction was monitored and electronically logged for each of these environments, which then provided us with user-navigational pat-terns taxonomy. Descriptive and statistical tests were carried out on the activities ob-served within this taxonomy to develop a framework for comparing the reading patterns for readers working in individual and document sharing environments. This framework was then used to obtain user feedback during the interview sessions that were held with the participants of this study. Because of our investigation, we were able to create a set of specific recommendation that system designers can use in order to create better, more intuitive, and user-friendly electronic reading and marking systems.

Introduction 
literary and historical scholars (Coleridge, 1985; J ackson, 2001; Stoddard, 1985; Tribble, 1997), by scholars in the field of educational psychology, (Fowler \& Barker, 1974; Idstein \& Jenkins, 1972; Nist \& Kirby, 1989), by researchers focusing on collaborative authoring settings (Adler, Gujar, Harrison, O'Hara, \& Sellen, 1998; Neuwirth, Kaufer, Chandhok, \& Morris, 1990) and finally by computer scientists for the design of computer based text reading and marking applications (Marshall, 1997; Wolfe \& Neuwirth, 2001).

As more and more documents can be found only in their digital form (Brush, Bargeron, Gupta, \& Cadiz, 2001), the issue of annotating and marking documents in the electronic environment becomes increasingly important. Interest of the research community towards marking e-documents is visible in several studies that explore the concept of paper document meta-phor - the imitation of the paper-based or "physical" marking experience, which includes marking a document by using a tablet PC and an intelligent pen-based device (Golovchinsky \& Denoue, 1999; Golovchinsky, Price, \& Schilit, 1999; Gotze, Schlechtweg, \& Strothotte, 2002). Other marking formats have been proposed; for example, the notion of cohabitation between the electronic and Paper Augmented Digital Documents (PADDs) (Guimbretiere, 2003), or the con-cept of multivalent documents.

The premise that computation enhances the process of active reading ${ }^{1}$, a necessary skill for professional and personal success, requires that we need to learn more about how users interact with applications offering electronic (e-) document marking possibilities. To date, only brief and partial attempts (Cadiz, Gupta, \& Grudin, 2000; Churchill, Trevor, Bly, Nelson, \& Cubranic, 2000; Marshall \& Brush, 2002, 2004; Marshall, Price, Golovchinsky, \& Schilit, 2001) have been made to characterize and quantify the elements of markings placed on electronic documents or to study the user's online reading and navigational activities accompanying them. The focus has been on the kind of information marked, and the underlying system architecture and technologies of the applications for marking e-documents (Guimbretiere, 2003; Gotze, Schlechtweg, \& Strothotte, 2002). There are no investigations that directly go towards explaining how users inter-act with electronic annotating applications while reading e-documents, especially with an eye to-wards informing the design of new technologies. The over-riding assumption in previous studies and system development efforts has been that when interacting with electronic applications, us-ers will behave in the same manner as they do when interacting with paper documents. Such an assumption is not necessarily correct (Gotze, Schlechtweg, \& Strothotte, 2002; O'Hara \& Sellen, 1997) and perhaps it is one of the reasons why so few users are actively using electronic marking applications. We intend to fill this knowledge gap. This aspect of exploring active reading is es-sential as we head towards an age of digital libraries, because the cyber users will now be able to browse electronic documents while crossing the boundaries between reading and 
editing. There-fore, a successful and user-friendly digital library interface design will be the one that can facilitate this new role of library patrons.

\section{Methods and approaches \\ Study objectives, task, and instruments}

The purpose of this study was to explore the interaction between graduate students and elec-tronic reading applications, which also function as electronic writing platforms, to understand the navigational structure of user reading and markings activities for sensemaking purposes. To achieve this research purpose, reading and marking navigational activities were catalogued while the electronic (e-) documents were being read, and possibly marked. This data was then ana-lyzed statistically and qualitatively along with survey and intenview data to seek, examine, dis-cover, identify, and categorize the significant interactions, correlations, themes, and sense making patterns.

The investigation was carried out in two types of electronic environments. In the first case, readers read the electronic documents individually or in a private environment, while the second was a document sharing environment, where the read documents were passed onto to another user. Graduate students were chosen as the target population in this study. This choice was in-fluenced by the fact that graduate students are required to read research articles as part of their course work. The participating students were all enrolled in the same course, and were paid a compensation for taking part in this project.

The task given to the participants was that they would be reading the e-articles to self inform, and gain sufficient knowledge so that they can lead a class discussion. This type of reading task was deemed ideal for this study as it is relatively slow and demanding, and also involves reflec-tion, memorization and testing of understanding (O'Hara, 1996). This task was also chosen be-cause research showed that readers annotate more if they are expected to actively participate in the discussion rather than simply sit in one (Marshall, Price, Golovchinsky, \& Schilit, 1999; Wolfe, 2001).

Adobe's Acrobat ${ }^{T M}$ was the main instrument for this study and all participants, novices or ex-perts were required to read and mark electronic documents (in PDF-Portable Document Format) by specifically using this software. Each participant was given 20 minute 
training in the usage of Acrobat's ${ }^{\mathrm{TM}}$ marking and navigational tools. Simultaneously, user interaction with Acrobat was observed and logged by using Lotus/ IBM's ScreenCam ${ }^{\mathrm{TM}}$. Factors measured included user read-ing and browsing patterns, for example, the readers skim, scan, read, skip and reread a docu-ment or an annotation within that document (Marshall et al., 1999). Also observed were the marking tools usage (tool switching, number of tools used etc.), and the marking patterns (includ-ing addition, editing or deleting behavior, re-annotating, responding).

The recorded observations were then used in the interview sessions, which were held with each participant soon after their last reading. The intenviews were held at the end rather than after each reading so that the investigator could study all the video logs, and establish the participant's navigational and marking patterns before the intenview. This theme identification then led to an effective exploration of the user's cognitive processes, as the participants responded better to queries probing their general usage, rather than specific actions. The data collected from these intenviews helped in the development of the navigational functions framework.

\section{Research questions, hypothesis, and methodology}

Given the lack of research in this area, our study was an exploratory and baseline investiga-tion shaped around the following research question:

RQ: What are the key navigational activities of users in private and shared electronic read-ing/ marking environments, and what are the user-perceived functions of these activities?

Once a spectrum of these navigational actions has been identified, a null hypothesis was formulated in order to help answer the research question. The null form for deemed ideal for this inquiry, as there is no evidence of a similar earlier investigation.

$\mathrm{H1}$ : The mean scores of each of the navigational activities for the group in a private environ-ment will not differ significantly from the mean scores of activities for the group that worked in an asynchronous document sharing environment. 
To achieve the research objectives, this project was carried out by employing a between-subjects design where the participants were randomly divided into two groups, group I and group S. The treatment imparted (independent variable) during this experiment was the environment, while the navigational activities were the dependent variables. In this case, the treatment (envi-ronment) had two levels (Martin, 1991), reading in individual and document sharing setting, each of which was imposed on one of the two groups. This design was consistent with the post-test only, non-equivalent control group design in a quasi-experimental setting, where the control group is also regarded as one of the treatments so that both groups have one treatment each (Campbell \& Stanley, 1972).

The participants were instructed to actively read the three articles electronically in a time-frame that coincided with their course schedule. The articles had been randomly selected from the course reading list by the project investigator. Members of Group I read private, individually owned documents that were not shared, while the members of group $\mathrm{S}$ read documents by shar-ing them via circulation among the group members. Thus, all participants of group S were in-formed at the beginning of this study that any markings or comments they make during the read-ing sessions would be seen and read, and perhaps commented upon and/ or edited, by other par-ticipants. The two groups worked in parallel on this project during the course of one semester. The methodology followed by these two participating groups is illustrated in Figure 1. 


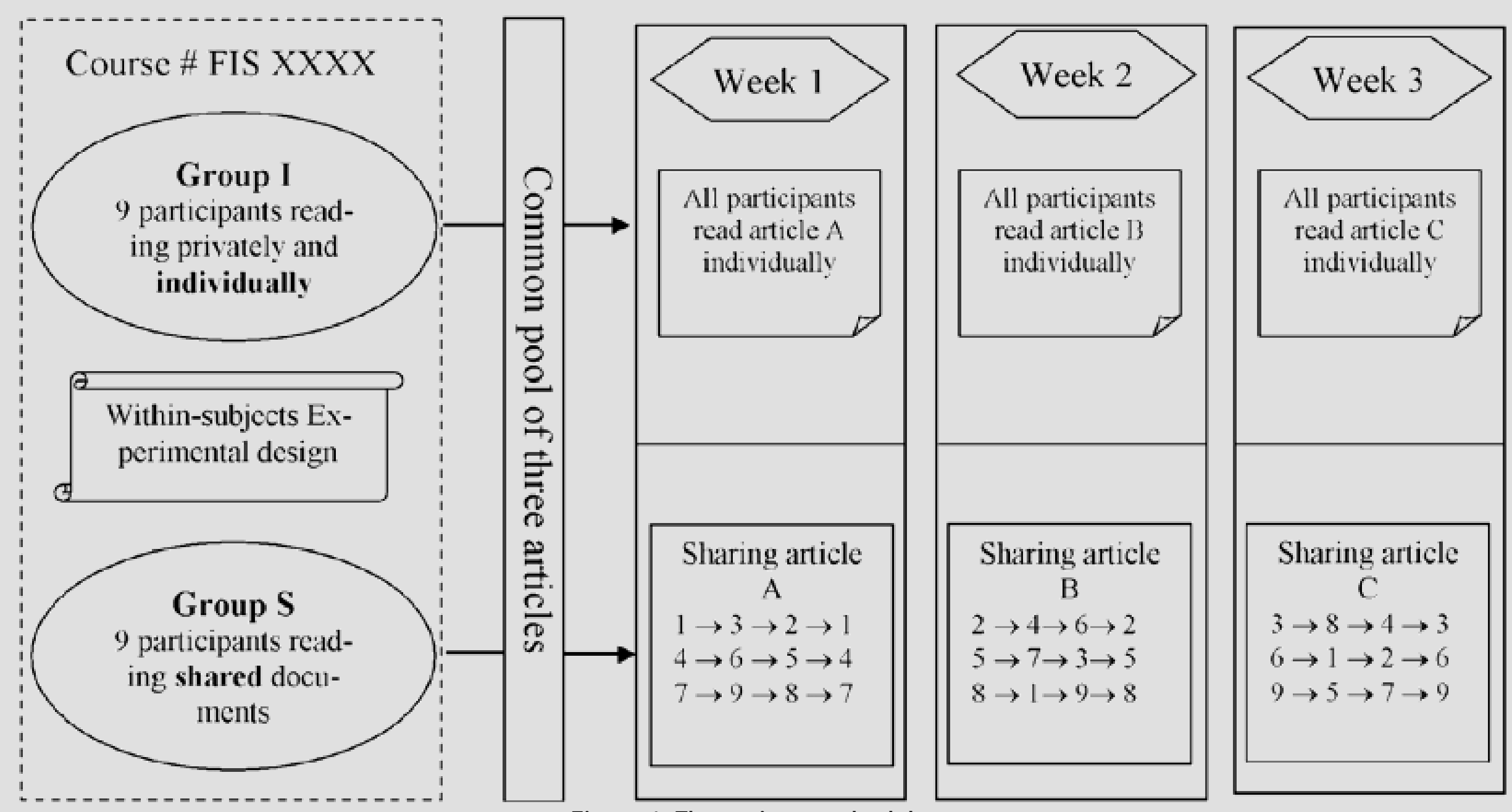

Figure 1: The project methodology

For group I, nine students were recruited and each student read the allocated three articles according to the weekly class schedule, thus generating 27 articles. The same three articles were read by the nine participants of group S, where each participant read those three articles in a document sharing environment, consisting of multiple rounds, as per the schedule shown in Figure 1. For example, in the first round, the three articles were randomly assigned to three members of group S so that users 1, $4 \& 7$ got Article A to read 
in the first round of Week 1 . The three read, and possibly marked, electronic articles obtained after the first round were reassigned to three other participants in the second round, and this trend continued till the fourth round where the initiating readers got to review the markings made by other participants, and had a chance to add, delete, or edit the markings. This concluded the shared reading schedule for Week 1 and the project moved into Week 2 and 3, with the only changes being made in initiating reader and the article.

All the experimental arrangements were designed to maintain the user's regular reading patterns and their natural settings so as to distance the researcher and research related aspects from the actual intensive reading process. To ensure this, the users undertook their readings alone, unaccompanied, in a public computer lab. After the experiment ended, interviews were held with each participant to understand their reading and markings activities, and to seek explanations for the functions of the navigational activities that the participants undertook.

\section{Results, analysis, and discussion}

Profiling our participants

Eleven females and 7 male graduate students took part in this study. They were divided ran-domly into two groups to form the individual and shared reading environments, and most of them were in the age bracket 26-35 years (50\%). This participant pool yielded 17 questionnaires, 60 edocuments (with 1,923 markings), 56 computer logs, and 16 inteniew transcripts. Responses on questionnaires indicated that almost all of the study participants used a computer daily, and ap-proximately $70 \%$ had more than five years of experience in using word processors. For graduate students, a surprisingly vast majority had little or no experience with the somewhat advanced fea-tures of word processors. Except for three participants, all others were familiar with, and had used Adobe's Acrobat $^{\mathrm{TM}}$. Very few people (35\%) indicated that they had shared annotated paper docu-ments, and they too reported an infrequent occurrence of this activity. However, a relatively high number of users reported that they had shared annotated electronic documents (60\%) with vary-ing frequencies.

\section{On-Screen activity observation}


User activities were monitored and recorded, and the observed interactions were analyzed to note the significant navigational and marking patterns, which are catalogued in Table 1 . The first observation was that when users engaged with an e-document in a private reading session then it was possible to predict an approximate area on which they were focusing, namely by tracking the movement of their mouse pointer on screen. This was because some users continuously moved their mouse along the lines being read. Similar movement was not often noticeable in the case of readers in shared setting. Our second observation was attained by assessing the screen-scrolling speeds, which indicated the areas of deep reading, skimming, or text skipping. While most of the deep reading seemed to have taken place in the beginning, the skimming usually took place when the readers scanned the reference list at the end, or when they encountered lengthy tabular text. In addition, readers moved back to read and mark more often compared to forward move-ments within the document, the latter being associated with reference checks. The total back and forth movement was not as much as one would expect for paper document readers, and occurred on average, only 1.7 times per document for individual readers and 2.1 times for document shar-ers. Other than this, the readers changed reading and marking properties infrequently, and these adjustments were usually observed in the beginning when the readers engaged in fine-tuning the reading environment.

Analysis of the tool usage showed that on average, each user used more than two marking tools ( $M=2.2$ (private), $M=2.5$ (shared)) during a reading, showing that readers were not interested in using more than 2-3 marking tools per reading session. However, readers switched tools quite often, especially when sharing documents ( $M=5.7$ (private), $M=13.4$ (shared)). In this con-text, a few noticeable instances, mostly in shared environments, occurred when users switched tools in the middle of marking a sentence, or when marking adjacent sentence ( $M=0.6$ (private), $M=3.2$ (shared)). Color changes were made only occasionally, on average, 1.6 times per docu-ment in case of individual readers, and 2.3 times per document in case of document sharing readers.

Not many instances were recorded under the "Other marking events" category. The only noteworthy event was that readers who shared e-documents often edited the old markings made by previous users. There were a few instances where readers edited their own markings, indicat-ing that markings once made were usually not altered. Placing multiple markings around the same text segment (varying combinations of anchors, annotations, and sidemarks) was also no-ticeable as it occurred on average, 1.4 times per document for individual readers, and 2.2 times per document in document sharing environments. 
We noticed that readers also lost a few markings that they had made while undertaking some follow-up activity, and then tried to nullify their actions by using the 'undo' command. However, the 'undo' command addressed not only the current focus but also some past actions, which re-sulted in the unintentional loss. It is possible that the availability of a certain warning mechanism could be helpful in preventing such mishaps from occurring.

Table 1: Testing for navigational differences between environments 


\begin{tabular}{|c|c|c|c|c|}
\hline e-Activity observation & $\begin{array}{l}\text { Individual } \\
\text { (Mean doc } \\
\text { participant) }\end{array}$ & $\begin{array}{l}\text { Shared docs } \\
\text { (Mean doc } \\
\text { participant) }\end{array}$ & $t$ & $\begin{array}{l}\text { Sig. } \\
\text { (p) }\end{array}$ \\
\hline \multicolumn{5}{|l|}{ Reading/Navigation activities } \\
\hline Mouse pointer indicates reading position & $\begin{array}{l}\text { Indicated some- } \\
\text { times }\end{array}$ & $\begin{array}{l}\text { Mostly not indi- } \\
\text { cated }\end{array}$ & & \\
\hline Skipping instances & 1.4 & 1.6 & 0.41 & 0.684 \\
\hline Moving back to reread/edit & 0.9 & 0.6 & -0.99 & 0.339 \\
\hline Moving back for rereading/marking & 0.7 & 1.3 & 0.82 & 0.823 \\
\hline Moving forward to read some advance text & 0.1 & 0.2 & 1.22 & 0.240 \\
\hline Change options/properties & 1.9 & 2.3 & 0.42 & 0.681 \\
\hline Use Other software (Internet, e-mail etc.) & 0.8 & 0.1 & -1.09 & 0.304 \\
\hline \multicolumn{5}{|l|}{ Usage of Marking tools } \\
\hline No. of times a marking tool was changed & 5.7 & 13.4 & 1.53 & 0.156 \\
\hline No. of different tools used & 2.2 & 2.5 & 0.63 & 0.539 \\
\hline Different tools used within 1-2 sentences & 0.5 & 2.5 & 1.63 & 0.137 \\
\hline Different tools used within one sentence & 0.1 & 0.7 & 1.12 & 0.293 \\
\hline Color changes made & 1.6 & 2.3 & 0.46 & 0.653 \\
\hline \multicolumn{5}{|l|}{ Marking events } \\
\hline Markings made inadvertently & 0.7 & 0.4 & -1.04 & 0.315 \\
\hline Delete/edit own markings & 1.4 & 1.8 & 0.71 & 0.487 \\
\hline Delete/edit previous markings & --.- & 9.1 & ---- & ---- \\
\hline Marking efforts fixed after facing problems & 0.3 & 0.3 & -0.18 & 0.857 \\
\hline Compound (Mark(s) + Annotation) & 1.4 & 2.2 & 1.44 & 0.184 \\
\hline No. of post reading markings & 0.2 & 0.2 & -0.14 & 0.891 \\
\hline No. of marking lost inadvertently & 0.1 & 0.0 & -1.51 & 0.169 \\
\hline No. of marking efforts abandoned & 0.3 & 0.4 & 1.20 & 0.249 \\
\hline Doing copy/cut and paste within document & 0.2 & 0.1 & -0.47 & 0.644 \\
\hline Place links within document & 0.1 & 0.0 & -1.00 & 0.347 \\
\hline
\end{tabular}


Our hypothesis looked for inter-environment differences within the listed activities in Table 1. To test this hypothesis, the use of the Levene's test for equality of variances in SPSS, was used and the last two columns of Table 1 include the results of the independent samples t-tests. These fig-ures were recorded after the F-statistic in Levene's test indicated the relevant $t$ values. The $p$-values indicate that none of the observed activities varied significantly within the two environ-ments. The only notable differences were that more frequent switching of marking tools $(t=1.63, p<0.15)$ was observed within sentences for collaborating users, and that sharing users tended to switch tools more often $(t=1.53, p<0.20)$. These differences were not statistically significant. Therefore, based on the evidence that was gathered, the hypothesis was accepted, or it can be said that the mean scores for the user's navigational activities for the e-document readers reading in a private environment do not differ significantly from the mean scores of the users working in a document sharing environment.

\section{Analyzing reading and navigational activities}

By obsenving the participants' reading and navigational behavior and comparing them with the reader feedback obtained during the interview sessions, the following patterns were immedi-ately noticeable:

One column format for e-reading

The readers almost unanimously voiced their strong reading preference for the one column format. This was true even for readers who mentioned that they preferred the two-column format for reading paper documents, "This article the format is very small and the layout not sepa-rated. I don't like two columns. Two columns always make me think the article is very long ... Even for the paper 
documents I prefer single column".

Mouse pointer as an indicator of attentive reading

When the movement of mouse pointer indicated the reading position of a reader, the reader was undertaking a concentrated and deep study of the e-document. The deep study was influenced by factors such as the section of the text, textual layout, work area, etc. For example, the move-ment was very noticeable in the two-column text layout, and at least five participants supported this assertion as is evidenced by the following comment: "This (2-column) kind of layout looks more professional, but it's hard to understand. So I don't remember, so I read it line by line".

Disregarding wordy illustrations

Lengthy and verbose illustrations such as the ones found in long tables were generally ignored, as was observed in this study where more than $80 \%$ of the readers either briefly skim the long tables or ignore them all together. Four participants commented on this, and one mentioned: "I think I remember that [the long table]. And I didn't find it very helpful ..."

References for research readers

Workers undertaking a self-informative reading task such as the one in this study ignored citations and reference lists. Almost all readers ignored the reference lists. When questioned about it, most simply replied that they would go through the references only while doing some research work, while others stated that this was the way how they read documents and it did not matter what medium was used: electronic or a traditional paper-based: “I didn't feel the need to read everything. I knew they [references] were attached to the document. So I need to go back, I know where they are. At this point, I don't have enough background to assess what 
I am looking at..."

\section{Memory triggers}

Three readers went back and read/ marked text that was triggered by something further along. Thus, only a few instances of rereading/ remarking were witnessed, which showed the user unease with electronic spatial movement One participant noticed: "I sometimes highlight as I read but then maybe if I don't understand or if the paragraph is about a whole idea so then I would like to read it first and then see what the main points were..."

Dictionary as a component of reading applications

The only Internet use observed during the study was when three users visited a web site to obtain meaning or translation for a word or a term. This is reflected in the following comment: "Ever since the introduction of Internet, I have been very dependent [on the online dictionary]. Because I think it's faster to look up words than a paper dictionary".

Marked sections read first, and faster

While obsening the reading behavior of e-documents, we noticed that at least four users out of nine in the shared reading condition read faster on the pages that were heavily marked. When asked about it, some readers agreed stating that they would likely read marked portions of a document first, especially those closer to the top of the page: "What I did was that I just ran through the marked ones first... Then I relate it to the paragraph and if it is important then I don't care. If it is missing meaning then I underline".

\section{Usage of the Marking tools}


A significant activity that was observed in the study was the frequency with which readers switched tools to mark. A look at Table 1 shows that on average, users switched tools almost six times when reading privately, but switched more often $(M=13.4)$ when reading a previously marked article. Similarly, the number of tools used was also significant but relatively stable across environments (private $-M=2.2$, shared- $M=2.5$ ). Also seen were relatively high instances of tool switching between sentences, or even within a sentence, where users marked half a sentence with one tools and the rest with another tool. Focusing on the tool usage characteristics, the fol-lowing main themes emerged from the user interviews:

Tool switching not distractive

The tool switching statistics were contrary to general perception, which assumes that tool switching is distracting and diminishes seamless reading. When three heavy switchers were questioned about their tool changes, the responses were that even on paper, they would need to reach out and pick up a highlighter and then switch to a pen depending on their marking needs: "I didn't really find it (tool switching) distracting like if wanted my main points to be highlighted and the sort of supporting points to be underlined. If I was looking at it on paper, I would probably have my highlighter and pen and I would probably change anyway"

Idea splitting

When users encountered two different ideas or two supporting ideas within a sentence or close together in the form of two sentences perhaps, they sometimes used two different colors or two different types of marking tools. This seemed mostly a subconscious decision and most readers did not remember the exact function at the time they performed the act. This trend is indicated by the following remark: "I can't remember exactly but I think it could be because that sentence might have a main idea and then some supporting idea". 
While the above-mentioned trends were more associated with readers in document sharing environments, individual readers tended to be much more conservative in switching tools too often or using the various types of tools available to them: "I think it was just the one [tool] that I was most familiar. That I have used before. I guess I wasn't that curious".

\section{Marking Events}

The last of this e-Activity qualitative analysis dealt with observed marking events such as de-leting or editing markings made by self or others, when the users placed different types of mark-ings close to each other, and other minor events. The following themes were observed in this category:

\section{Editing markings when facing software problems}

This aspect was noticed when the users struggled to familiarize themselves with the working environment in both individual and collaborative situations. Table 1 showed that users made many inadvertent markings, and some users made a visible effort of resolving the problem they faced. Some managed to get it right, while the others simply gave up. This remained true in document sharing situations as most of the editing that took place on old markings occurred because the reader faced some kind of problem, the most common being the abnormal highlight-ing color. Otherwise, most said that they preferred not to change the previously made markings unless they were obstructing the vision, or causing any other hindrance. For example, a reader remarked, "I thought it was messy and I didn't think it was as catchy as the word I was interested in. Also, after I read the paragraph, I think I went back because this deserved a bit more focus". 
Sharing readers worked amid old markings to create their desired relationships by compounding markings (placing more markings over the older ones). This was similar to the creation of virtual relationships that were observed earlier in the case of compound markings made by private readers. These relationships are between sentences, paragraphs, or other points of interest. This is apparent from comments such as: "Well, I highlighted (over old highlighting) to provide a relationship with the next sentence".

Multi-marks for visibility

Among the clutter of markings, users felt that they had to place side marks in addition to an anchor, or another type of mark to place emphasis on a particular point, and thus make it stand out. This multi-marking scheme was especially useful in the case of users who expected to flip through the article in the future and quickly grab the key points. This assertion holds true for both the environments, and the readers supported this through remarks such as: "I think I underlined first and then I put the note in the margin. Like putting in this asterisk in the margin. So if I was flipping though things, this would be something I would look at a bit more." A special type of multi-mark would be the one indicating an error. This kind of common marking strategy was observed in case of some of the more sharpeyed readers who noticed a typo and circled it. They then proceeded to place a question mark besides it.

\section{Document marking system design}

Based on the quantitative and qualitative analysis of the data gathered from this text marking activity investigation, some key issues emerged that affect the design of the user interface, and, may require additional algorithms. We have summarized some of these key requirements here. 


\section{e-Document layout and design}

Investigations into reader perception of the issues of the design of electronic reading applica-tions, which allow users to act as authors by marking up the document, reveal a lack of user sat-isfaction with the current reading layouts. Two column layouts head the list of items that require a change in the e-environment as the format was deemed highly reader unfriendly. Conversely, using a bigger font was not that much of an influencing factor as users adapted by increasing or decreasing the screen view size. Other than these, the default user view area should show the page with visible side borders so that the users get a better sense of the spatial layout of the page.

Lengthy textual content placed in tables is usually skimmed or skipped altogether, as readers perceive it to be a repetition of what is in the article itself. Therefore, journal authors should be encouraged to place more graphic and illustrative data in figures to support and promote online reading. Additional text, such as author bios or commentaries should be placed before the refer-ence list as the readers lack the spatial sense associated with paper documents and are thus liable to miss those extra textual items when they skip references. The reference lists themselves should be hyperlinked to the in-text citations to encourage more hits, and could be placed in an on-demand hidden area for readers who need them, or for readers whose profile identifies them as researcher readers.

Placing bookmarks to cater for memory-triggered navigation should be as easy as placing a highlight so that the readers do not fear getting disoriented or lost in the cyberspace when they move back to reread. Complex implementations of this feature, such as the one in Acrobat, dis-courage back and forth movements within the e-document. Finally, the addition of a dictionary, and perhaps a thesaurus, is a necessity for any e-book reading software.

\section{Marking tools design}

Users prefer to use only a few marking tools. Therefore, user profiles can be customized to present each user with a personal 
marking toolbox, containing only the most recent and frequently used tools. The rest of the options can be hidden initially, but should remain conveniently accessible through dropdown lists or expandable tool bars.

Colors can be managed in a similar manner, as most users tend to make use of only one or two colors during a reading session. Similarly, the default highlighting color should be such so as not to obscure text, or is a preferred color based on the reader's usage profile.

As this research indicated, individual readers tend to switch tools less often. Visual aids should be provided to readers so that they can keep track of their in-use marking tool. This can be implemented by ensuring that an icon of the selected feature is visible with the mouse pointer so that the reader sees the marking instrument in "hand" without taking the eyes off the reading area.

\section{Application design for sharing document markings}

The user responses regarding the issue of starting with a clean document or a marked one were heavily tilted in favor of reading a clean document. Thus, as first time readers generally pre-ferred to start with a clean, unmarked document, most readers thought that this was the way an e-document should be offered initially for viewing. Additional layers of markings could then be made available so that the reader may superimpose any one, or more of the layers on that docu-ment. User response also indicated that readers were more likely to place emphasis, or deemphasis, on markings from a familiar person, and thus marking layers containing markings from known users would be preferred, or not preferred as the case may be.

Within the markings, annotations were the most sought after because they help explain, clar-ify, and summarize the original text. This reflection is in harmony with this research's earlier find-ings, which showed that the occurrences of short sentences and notes went up in document sharing situations. The reader reactions also showed that attitudes towards markings other than annotations was not very positive, and most users would like to see minimal amounts of highlight-ing, and even less of the other types of markings. However, despite the negative attitude towards markings, most of the readers in this study were reportedly comfortable with the old markings and did not view them all overly distracting. In fact, some of the users commented that appropriate placing of markings did function as knowledge adding facets. 
Most readers were comfortable about putting their names and their annotations as long as the sharing took place within a known group of people. However, marking a public document was not seen in a positive light. Finally, a sharp rise in the base marking types or anchors, such as, underlining and highlighting, occurred in collaborative situations as readers tried to improve the visibility of their markings and that of the relationships that they created.

\section{Limitations}

Our study had several limitations. First, we were limited to the use of one computer applica-tion exploring the interactions. This was felt necessary to ensure that a common environment and a universal set of marking tools would be available to all readers. A second limitation was that through the introduction of the active reading process using an electronic reading/ editing system, we intervened in a user's regular reading practice that normally consisted of taking a printout of an electronic article and reading it on paper. Lastly, we conducted this study on a limited number of student readers reading for a specific task. A different task in a different setting with a larger participant pool may influence the results. We realize that while these limitations do restrict the generalizability of this study, the results do satisfactorily portray the text marking activity patterns of knowledge readers accessing electronic documents.

\section{Conclusions}

In this study, we studied the user activities that took place when a reader read a short journal article in electronic format and possibly marked them using a set of available marking tools. Sig-nificant activities were noted, and were then utilized in getting user feedback to gain insight into their perceptions of marking activities. We strongly believe that the identified guidelines for read-ing layout, marking tools design, sharing and navigational experiences can be used by system designers for improving the design of future electronic reading and marking applications. 


\section{Notes}

IIndicates reading activity where the reader acts an author, and engages in writing on the text while reading, or takes notes in a separate notebook.Back

\section{References}

Adler, A., Gujar, A., Harrison, B. L., O'Hara, K., and Sellen, A. (1998, Apr. 18 - 23). A diary study of work-related reading: design implications for digital reading devices. Proceedings of the Conference on Human Factors in Computing Systems, Los Angeles, CA.

Brush, A. J. B., Bargeron, D., Gupta, A., and Cadiz, J. J. (2001). Robust annotation positioning in digital documents. Proceedings of the SIGCHI '01, Seattle, WA.

Cadiz, J. J., Gupta, A., and Grudin, J. (2000, Dec.). Using web annotations for asynchronous collaboration around documents. Proceedings of the CSCW '00, Philadelphia, PA.

Campbell, D. T., and Stanley, J. C. (1972). Experimental and quasi-experimental designs for research. Chicago: Rand McNally \& Co.

Churchill, E. F., Trevor, J., Bly, S., Nelson, L., and Cubranic, D. (2000, Apr. 1-6). Anchored conversations: chatting in the context of a document. Proceedings of the CHI 'OO Conference on Human Factors in Computing Systems, The Hague, Netherlands.

Coleridge, S. T. (1985). Marginalia (Vol. 12, pt. 1-6). Princeton: Princeton University Press.

Fowler, R. L., and Barker, A. S. (1974). Effectiveness of highlighting for retention of text material. Journal of Applied Psychology, 59(3), 358-364.

Idstein, P., and Jenkins, J. R. (1972). Underlining versus repetitive reading. Journal of Educational Research, 65, 321-323.

Jackson, H. J. (2001). Marginalia: Readers writing in books. New Haven and London: Yale University Press. 
Marshall, C. C. (1997, July 23-36). Annotation: from paper books to digital library. Proceedings of the 2nd International Conference on Digital Libraries, Philadelphia, PA.

Marshall, C. C., and Brush, A. J. B. (2002, April 20-25). From Personal to Shared Annotations. Proceedings of the CHI 2002 Conference on Human Factors in Computing Systems, Minneapolis, MN.

Marshall, C. C., and Brush, A. J. B. (2004, June 7-11). Exploring the relationship between personal and public annotations. IEEE/ACM Joint Conference on Digital Libraries, Tucson, AZ.

Marshall, C. C., Price, M. N., Golovchinsky, G., and Schilit, B. N. (1999, Aug. 11-14). Introducing a digital library reading appliance into a reading group. Proceedings of the Fourth ACM International Conference on Digital Libraries, Berkeley, CA.

Marshall, C. C., Price, M. N., Golovchinsky, G., and Schilit, B. N. (2001, June 24-28). Designing e-books for legal work. Proceedings of the ACM/IEEE Joint Conference on Digital Libraries, JCDL '01, Roanoke, VI.

Martin, D. W. (1991). Doing psychology experiments (3rd ed.). Pacific Grove, CA: Brooks/Cole Publishing Co.

Neuwirth, C. M., Kaufer, D. S., Chandhok, R., and Morris, J. H. (1990, Oct. 7-10). Issues in the design of computer support for co-authoring and commenting. Proceedings of the Conference on Computer-supported Cooperative Work, Los Angeles, CA.

Nist, S. L., and Kirby, K. (1989). The text marking patterns of college students. Reading Psychology, 10, 321-338.

O'Hara, K. (1996). Towards a Typology of Reading Goals (Technical Report EPC-1996-107). Cambridge, UK: Rank Xerox Research Centre, Cambridge Laboratory.

Schilit, B. N., Golovchinsky, G., and Price, M. N. (1998, Apr. 18-23). Beyond paper: Supporting active reading with free form digital ink annotations. Proceedings of the $\mathrm{CHI}$ '98 Conference on Human Factors in Computing Systems, Los Angeles, CA.

Stoddard, R. E. (1985). Marks in books, illustrated and explained. Cambridge, MA: Harvard University Press. 
Tribble, E. B. (1997). "Like a looking-glass in the frame": From the marginal note to the footnote. In D. C. Greetham (Ed.), The margins of the text (pp. 229-244). Ann Arbor, Ml: University of Michigan Press.

Wolfe, J. L. (2001). Pedagogical uses of annotations and annotation technologies. Unpublished PHD Dissertation, University of Texas, Austin.

Wolfe, J. L., and Neuwirth, C. M. (2001). From the margins to the center: The future of annotations. Journal of Business and Technical Communication, 15(3), 333-371. 